\title{
The effect of unequal strand length on short DNA duplex hybridization in a model microarray system: A Monte Carlo simulation study
}

\author{
Sarah J. Cooper, John M. Stubbs* \\ Department of Chemistry and Physics, University of New England, 11 Hills Beach Road, \\ Biddeford, ME 04005
}

\begin{abstract}
Monte Carlo molecular simulations of DNA decamer-hexamer strands were carried out over a range of temperatures, both free in solution and bound to a surface in several possible fashions, to investigate the effect of dangling end nucleotides on hybridization. For comparison, analogous hexamer duplexes were also studied in solution. Duplex stability was analyzed in terms of energetic, hydrogen bonding, and structural properties. Of the systems studied, shorter solution sequences interacting with longer surface-bound sequences generally had weaker interactions than the opposite.

Key words: DNA, Hybridization, Microarray, Dangling End, Monte Carlo, Simulation
\end{abstract}

\section{Introduction}

A DNA microarray is a system consisting of single strands of DNA, called probes, bound to a solid surface that will ideally hybridize with their comple-

*Corresponding author. Fax: +1-207-602-5993

Email address: jstubbs@une.edu (John M. Stubbs)

Preprint submitted to Chemical Physics Letters

June 10, 2015

(C) 2015. This manuscript version is made available under the Elsevier user license 
mentary strands, known as targets, from solution. Typically the probe strand is a known sequence, allowing it to function as a sensor to detect the presence of a labeled target from a sample since hybridization with other, closely related, sequences is generally not as stable as with the perfect complement. DNA microarrays are useful for studying mutations, polymorphisms, and other areas of interest including detection and characterization of microbial pathogens, and genotyping $[1,2]$.

The introduction of a surface has been seen to influence the hybridization interactions of target and probe strands to one another $[3,4,5,6]$. Further complicating the issue is that often experimentally the probe and target strands are of different lengths which results in the presence of a dangling end, or extra nucleotides, that do not have a complement on the other strand on one or both ends [7]. In solution, dangling ends for short DNA duplexes have been shown to be generally stabilizing, although not in all cases $[8,9]$; it is also less pronounced than the analogous stability effects for RNA $[8,10]$. One recent study looked at the experimental effect of having a dangling end and found that stabilization is in proportion with the level at which the dangling nucleotide has stacking interactions with the neighboring, hydrogen bonding, hybridized base pair [11]. The identity of the nucleotides in the dangling end have also been shown to contribute to the effect $[8,11]$. Turning to microarray systems, Peytavi and coworkers [12] discuss that short (ca. 20 bases) probe strands, while being able to detect mismatches (i.e. mutations) in the sequence, have variable ability to discriminate between targets depending upon how a longer (ca. 400 bases) target's dangling ends are oriented, seeing greater hybridization efficiency when the target's dangling end 
is primarily at the surface proximal end of the probe-target duplex; more recently Tomlinson et al. found similar results [13].

Because of the additional complexities involved in surface-bound DNA hybridization, the study of these systems with predictive models is key to shedding light on the underlying physico-chemical properties $[14,15]$. While much of the previous work in simulation studies of DNA duplexes has been focused on strands of equal length, recent work has corroborated the idea that the dangling end nucleotide's interaction with neighboring hydrogen bonded bases is the reason for the differences in solution duplex stability between sequences with single nucleotide dangling ends [16]. Schmitt et al. [17] recently carried out simulations to study a 20 base probe and 60 base target in three different systems with a 40 base dangling end either at the surface distal end of the duplex, at the surface proximal end of the duplex, or with a 20 base dangling end on both ends. They calculated that all three hybridization motifs were similarly destabilized with respect to no dangling end(s) on the target. This group's previous work [18] on 11 base pair duplexes on a surface with Monte Carlo molecular simulation found two different effects on duplex stability due to surface binding: compared to the duplex in solution, nucleotides at the surface proximal end had destabilized hydrogen bonding, while those at the surface distal end had enhanced hydrogen bonding. Both effects were due to changing amounts of duplex end fraying, with an increased amount at the proximal end and decreased amount at the distal. With all this as background, the goal of the current work was to study model DNA microarray systems with short sequences of unequal length to characterize the effect of having a dangling end on DNA hybridization and stability, as 
well as to investigate differences among varying surface binding setups.

\section{Methodology}

\subsection{Molecular Models}

A coarse-grained representation of DNA is employed. Originally developed by Schatz and coworkers [19] and modified by our group, the model includes one interaction site to represent the sugar and phosphate groups and one site to represent the base functionality, with structural parameters (e.g. equilibrium bond lengths) chosen to represent the canonical B DNA structure and dispersion-repulsion and hydrogen bonding interaction parameters chosen to reproduce estimated denaturation temperatures. A complete description is available in our previous work [18].

\subsection{Simulation Details}

The sequences and system studied, depicted in Figure 1, are based on the decamer sequence

\section{5' CCAACTTCTT 3' \\ 3' GGTTGAAGAA 5'}

which was chosen because it had been included in our previous study of decamer mismatch and surface spacer length effects [20]. In order to investigate the effect of a dangling end, i.e. architectures where one strand is longer than the other, the modifications studied here are based on truncating the lower strand (strand 2) to six nucleotides in three different ways: the left-most, 3' GGTTGA 5'; the middle, 3' TTGAAG 5'; and the right-most, 3' GAAGAA 5'. Each of these decamer + hexamer duplexes were studied in five different 
environments: with both strands free (solution), or with either strand 1 or 2 immobilized on a surface, tethered with a five unit spacer, at either the 5' or 3' end. For comparison, duplexes without dangling ends (hexamer) were also studied in solution for each of the three sequences. Note that all surface 1 setups have the same probe strand as do all surface 3 setups.

Monte Carlo (MC) molecular simulations were carried out for each system in the canonical $(N V T)$ ensemble. In addition to the usual translation and rotation moves, the conformational degrees of freedom were efficiently sampled using coupled-decoupled configurational-bias MC [21, 22, 23, 24, 25] and self-adapting fixed-endpoint configurational-bias MC [26]. At least $2.5 \times 10^{8}$ and $1.25 \times 10^{9}$ cycles were used for equilibration and production, respectively, where $1 \mathrm{MC}$ cycle $=N_{\text {molecule }}=2 \mathrm{MC}$ moves. Each system was studied with a single duplex in either a $100 \AA \times 100 \AA \times 100 \AA$ cubic simulation cell for solution systems or $100 \AA \times 100 \AA \times 114 \AA$ rectangular simulation cell with a hard (i.e. purely repulsive) surface from $0<z<14 \AA$. Periodic boundaries were employed [27] as was a $14 \AA$ interaction distance cutoff. Temperature values included 240, 260, 280 and $300 \mathrm{~K}$, and were chosen so that each system goes from predominantly double-stranded to predominantly single-stranded over this range, i.e. the duplex undergoes denaturation. Statistical uncertainties were estimated from $5 \times 10^{7} \mathrm{MC}$ cycle block averages of the production data. A portion of this work was carried out with XSEDE supercomputing resources [28]. 


\section{Results and Discussion}

\subsection{Energetics}

The average total energy of hydrogen bonding, $U_{\mathrm{HB}}$, for each system as a function of temperature is given in Table 1. As expected, each duplex goes from predominantly hydrogen bonded at $240 \mathrm{~K}$ to predominantly dissociated at $300 \mathrm{~K}$ as indicated by the changes in $U_{\mathrm{HB}}$ values. For a given temperature and sequence, comparison between the different systems shows the effect of dangling ends and / or surface binding mode on the stability of the duplex.

For all sequences, comparing solution $U_{\mathrm{HB}}$ values to those of the hexamer, i.e. with and without the dangling end(s) shows only small differences, especially at the lowest temperature, considering the estimated uncertainties. Although for the middle sequence solution has slightly weaker $U_{\mathrm{HB}}$ values while for the right sequence it has slightly stronger values, the differences are not large. Comparing surface-bound results to solution values yields several trends. For the left sequence, surface 1 is consistently destabilized compared to solution while surfaces 3 and 4 are either comparable or slightly more energetically favorable than solution. For the right sequence, surface 3 is consistently destabilized with respect to solution whereas surfaces 1 and 2 are either comparable or slightly more energetically favorable. These are the same trends for both left and right due to the location of the dangling end and which strand (short or long) is surface bound. Because of the setup and sequence differences, left surfaces 1,2, 3, and 4 are analogous to right surfaces $3,4,1$, and 2, respectively. Thus it appears that systems where the target is shorter than the probe and the region of complementarity is at the proximal end while the dangling end is at the distal end (e.g. left surface 
1 ) is a generally destabilizing situation. On the other hand, systems with a shorter target that have the complementary region distal to the surface and the dangling end proximal (e.g. left surface 3) enhances interactions between strands and stabilizes the duplex. Interestingly, systems with a longer target than probe have comparable stability to solution for either the case of the dangling end at the surface distal or proximal end of the duplex. While the former is not surprising the latter is; it should be pointed out that for the systems investigated here, left surface 4 and right surface 2, the duplex's surface proximal dangling end of four nucleotides is shorter than the probe-surface five unit spacer so that duplex formation is not overly conformationally inhibited by the surface. It is doubtful that the same enhanced interaction would be present if the dangling end were longer than the spacer, which would force part of the target away from space already occupied by the surface.

For the middle sequence, surfaces 2 and 4 have comparable $U_{\mathrm{HB}}$ values to the solution results, whereas surfaces 1 and 3 are both less stable. This indicates that systems with short probes together with targets having a small number of dangling end nucleotides on both ends are markedly more stable than the opposite situation. In considering all three sequences and all surface setups, systems with short targets are more often than not destabilized with respect to solution $U_{\mathrm{HB}}$ values.

\subsection{Hydrogen Bonding}

In order to study the effects of surface binding and of having a dangling end more closely, the extent of hydrogen bonding for each individual nucleotide is determined. A given base is considered to be hydrogen bonded if its energy of hydrogen bonding exceeds the threshold value of $200 \mathrm{~K}$, i.e. 
if $u_{\mathrm{HB}} / k_{\mathrm{B}} \leq-200 \mathrm{~K}$ where $k_{\mathrm{B}}$ is Boltzmann's constant. This definition has been used previously with this model and has been shown to be reasonable $[20,18]$.

Figures $2 \mathrm{a}$ and $\mathrm{b}$ show the average number of hydrogen bonded base pairs, $N_{\mathrm{HB}}$, as a function of temperature for the left and middle sequences, respectively. As temperature increases all systems undergo denaturation into single stranded forms, and at any given temperature setups with a higher $N_{\mathrm{HB}}$ are more hydrogen bonded and have enhanced stability. For the left sequence, at most temperatures surfaces 3 and 4 have the highest $N_{\mathrm{HB}}$ values, with solution, hexamer and surface 2 slightly lower while surface setup 1 has the lowest values. This is consistent with the stability rankings from considering the total hydrogen bonding energy as described above; results for the right sequence are analogous (not shown). The middle sequence has surfaces 1 and 3 with fewer hydrogen bonds and all other systems fairly comparable, also consistent with relative stabilities from $U_{\mathrm{HB}}$ values described above.

Knowledge of an individual base's hydrogen bonds allows the determination of the fraction of configurations that it's participating in hydrogen bonding, which in turn allows the hydrogen bonding pattern of a duplex to be analyzed in detail. Figures $3 \mathrm{a}$ and $\mathrm{b}$ show the fraction of hydrogen bonding configurations for a given base for strand 1 of the left and middle sequences, respectively, at $240 \mathrm{~K}$. Base index numbers shown are the same as those in Figure 1 and are consistent across all systems, i.e. bases 1 and 2 for the left sequence are always the two adjacent cytosine sites in the decamer sequence. Comparing both sequences, the impact of GC content is apparent, with the left sequence, at 50\% GC pairs, having generally higher 
$f_{\mathrm{HB}}$ values for all bases than the middle sequence, at $33 \%$ GC pairs. For the left sequence, although the hexamer has intermediate values for most bases, for base 6 it has a higher value than all other systems. As base 6 in all other systems is adjacent to the 4 nucleotide dangling end, this indicates that the presence of this dangling end destabilizes the hydrogen bonding of the base immediately adjacent to it. Surfaces 1 and 2 have $f_{\mathrm{HB}}$ values consistently lower than all other setups, particularly at bases 1 and 6 . The destabilization at base 1 in these cases is also what would be predicted from our previous results [18] which showed destabilization of hydrogen bonding for bases proximal to the surface binding end of the duplex. Surface 3 has the same $f_{\mathrm{HB}}$ values as solution with a higher value at base 1, indicating little difference in hydrogen bonding interactions upon surface binding for most bases and enhancement at the distal end. However, given our previous results, base 6 , at the proximal end, would be expected to be destabilized. That this does not appear to be the case indicates that the destabilization is absent or lessened due to the dangling end between the duplex and the surface binding site. Surface 4 , although destabilized at base 6 , has some of the highest $f_{\mathrm{HB}}$ values for the first two bases, indicating both proximal destabilization and distal enhancement. An analysis of $f_{\mathrm{HB}}$ for the right sequence, not shown, yields the same trends.

The values of $f_{\mathrm{HB}}$ for the middle sequence have several interesting features. The lowest values at either end-surface 1 for base 3 and surface 3 for base 8-correspond to bases proximal to the surface when the probe is the longer sequence. Secondly, the highest values at either end (not including the hexamer)-surface 2 for base 8 and surface 4 for base 3-correspond to bases 
distal to the surface when the target is the longer sequence. Both of these results are consistent with the end effect findings discussed previously, and are also reflected in the general destabilization of the surface proximal bases such as base 3 for surface 2, which has $f_{\mathrm{HB}}$ values comparable or stronger than solution for every base except base 3. These effects also show up to a lesser extent as a slight enhancement of surface distal bases such as for surface 1 which comes closest to having the same $f_{\mathrm{HB}}$ value as solution at base 8 , though this is not enough to overcome the unfavorable destabilization at the other end. Thus, the stability of the various sequences and systems studied here are well described by our previous findings of distal enhancement and proximal destabilization, modified in that the proximal effect is lessened or absent in the case of additional probe nucleotides between the complementary region and the surface bound end.

\subsection{Structural Effects}

The double helix structure was examined for duplexes that were completely hydrogen bonded. The local helical axis was found by determining the midpoints between neighboring complementary base pairs, after which the rotation around that axis of adjacent base-backbone bond vectors was used to determine the base plane dihedral angle between neighboring base pairs, while the distance between midpoints was taken to correspond to the rise between base planes [18].

Changes in the resulting geometrical properties indicate the effect of the dangling end and of surface attachment on the duplex structure. Figures 4a and $\mathrm{b}$ give the base plane dihedral angles and rise values, respectively, for the left sequence at $240 \mathrm{~K}$. The structure of the hexamer is symmetric and has 
either end with a slightly higher dihedral angle by $\approx 0.7$ degrees and closer spacing by $\approx 0.06 \AA$ compared to the interior. Upon addition of the four nucleotide dangling end in solution, the end of the duplex adjacent to the dangling end has decreased dihedral angles and increased rise values more similar to values for the interior of the duplex. Out of the surface systems, surface 3 is most similar to these solution values where at the end of the duplex with the dangling end, base pairs 5-6, the values lie between the solution and hexamer values. Surface 4 is also similar, only deviating with a higher rise value for the pair next to the dangling end. As this pair is at the surface proximal end of the duplex, this increase may be due to the presence of the surface and / or spacer. Surface 2 has somewhat lower dihedral angles and higher rise values than most other helices but is not qualitatively different from setup 3, though the interior rise values at base pairs 3-4 and 4-5 are the highest of all the duplexes. Surface 1 is most different from all others, having little distinction between end and interior base pair values. Additionally, the dihedral angles are all up to 2 degrees lower and rise values up to $1.0 \AA$ A higher, indicating it's unwound and elongated compared to others, particularly at the ends of the duplex. In fact, the ends have some of the highest of all rise values while simultaneously having the lowest dihedral values.

Figures $5 \mathrm{a}$ and $\mathrm{b}$ give the base plane dihedral angles and rise values, respectively, for the middle sequence at $240 \mathrm{~K}$. The hexamer's values are similar to those of the left sequence; again, adding dangling ends lowers the dihedral twist angle and increases the rise values. Surfaces 2 and 4 also show this pattern but altered slightly to higher dihedrals and rise values at the proximal end compared to solution; as these two have $U_{\mathrm{HB}}$ values similar 
to solution these small perturbations must not have much of an impact on the overall duplex interactions. Surfaces 1 and 3, on the other hand, show very different effects. The dihedral is lowered by $\approx 0.8$ degrees from solution at the proximal end, much lower than other end pairs (though comparable to middle of 2 and 4 ) then steadily increases to the distal end. The rise value increases somewhat as well at the proximal ends, though not beyond values of other surfaces. Thus, for all systems, having a low dihedral angle at the duplex termini is related with destabilization of the duplex, so that helix underwinding is likely impacting the overall average hydrogen bonding energy.

\section{Summary}

Simulations of short DNA duplexes with varying dangling ends and surface binding motifs were carried out and analyzed via energetic, hydrogen bonding and geometrical properties. For the systems investigated here, the effects of a dangling end and surface binding were to increase base plane rise value and lower base plane dihedral angles. If addition of the dangling end on the probe increases the distance between surface and duplex, it also lessens proximal destabilization of hydrogen bonding; otherwise proximal destabilization and distal enhancement end effects both act on a duplex's stability. Taken together these results indicate that short targets generally have hybridization interactions the same or weaker than their solution analogs whereas longer target strands have the same or stronger interactions compared to solution. 


\section{Acknowledgment}

The authors wish to thank the University of New England for support through a sabbatical leave award and the National Science Foundation for support through grant number CBET-1264511. This work used the Extreme Science and Engineering Discovery Environment (XSEDE), which is supported by National Science Foundation grant number ACI-1053575.

\section{References}

[1] E.S. Lander, Nat. Genet. Suppl. 21 (1999) 3.

[2] R.B. Stoughton, Annu. Rev. Biochem. 74 (2005) 53.

[3] A.B. Ozel, O. Srivannavit, J.-M. Rouillard, E. Gulari, Biotechnol. Prog. 28 (2012) 556.

[4] H. Binder, J. Phys.: Condens. Matter 18 (2006) S491.

[5] R. Levicky, A. Horgan, Trends Biotechnol. 23 (2005) 143.

[6] A.E. Pozhitkov, D. Tautz, P.A. Noble, Brief. Funct. Genomics Proteomics 6 (2007) 141.

[7] A. Ferrantini, E. Carlon, Gene 422 (2008) 1.

[8] S. Bommarito, N. Peyret, J. SantaLucia Jr., Nucleic Acids Res. 28 (2000) 1929.

[9] B.G. Moreira, Y. You, M.A. Behlke, R. Owczarzy, Biochem. Biophys. Res. Commun. 327 (2005) 473. 
[10] T. Ohmichi, S. Nakano, D. Miyoshi, N. Sugimoto, J. Am. Chem. Soc. 124 (2002) 10367.

[11] J. Isaksson, J. Chattopadhyaya, Biochemistry 44 (2005) 5390.

[12] R. Peytavi, L. Tang, F.R. Raymond, K. Boissinot, L. Bissonnette, M. Boissinot, F.J. Picard, A. Huletsky, M. Ouellette, M.G. Bergeron, BioTechniques 39 (2005) 89.

[13] J. Tomlinson, C. Harrison, N. Boonham, S.A. Goodchild, S.A. Weller, BMC Res. Notes 7 (2014) 251.

[14] A. Halperin, A. Buhot, E.B. Zhulina, J. Phys.: Condens. Matter 18 (2006) S463.

[15] A. Harrison, H. Binder, A. Buhot, C.J. Burden, E. Carlon, C. Gibas, L.J. Gamble, A. Halperin, J. Hooyberghs, D.P. Kreil, R. Levicky, P.A. Noble, A. Ott, B.M. Pettitt, D. Tautz, A.E. Pozhitkov, Nucleic Acids Res. 41 (2013) 2779.

[16] M. Kara, M. Zacharias, Biopolymers 101 (2014) 418.

[17] T.J. Schmitt, B. Rogers, T.A. Knotts, J. Chem. Phys. 138 (2013) 035102-1.

[18] J.H. Allen, E.T. Schoch, J.M. Stubbs, J. Phys. Chem. B 115 (2011) 1720.

[19] K. Drukker, G. Wu, G.C. Schatz, J. Chem. Phys. 114 (2001) 579.

[20] N.B. Tito, J.M. Stubbs, Chem. Phys. Lett. 485 (2010) 354. 
[21] J.I. Siepmann, Mol. Phys. 70 (1990) 1145.

[22] J.I. Siepmann, D. Frenkel, Mol. Phys. 75 (1992) 59.

[23] D. Frenkel, G.C.A.M. Mooij, B. Smit, J. Phys.: Condens. Matter 4 (1992) 3053.

[24] J.J. de Pablo, M. Laso, U.W. Suter, J. Chem. Phys. 96 (1992) 2395.

[25] M.G. Martin, J.I. Siepmann, J. Phys. Chem. B 103 (1999) 4508.

[26] C.D. Wick, J.I. Siepmann, Macromolecules 33 (2000) 7207.

[27] M.P. Allen, D.J. Tildesley, Computer Simulations of Liquids, Oxford University Press, Oxford, 1987.

[28] J. Towns, T. Cockerill, M. Dahan, I. Foster, K. Gaither, A. Grimshaw, V. Hazlewood, S. Lanthrop, D. Lifka, G.D. Peterson, R. Roskies, J.R. Scott, N. Wilkins-Diehr, Comput. Sci. Eng. 16 (2014) 62. 
Table 1: Average energy of hydrogen bonding in $\mathrm{kJ} \mathrm{mol}^{-1}$. Subscripts indicate uncertainty in the final digit.

\begin{tabular}{lcccc}
\hline Left & $240 \mathrm{~K}$ & $260 \mathrm{~K}$ & $280 \mathrm{~K}$ & $300 \mathrm{~K}$ \\
\hline Hexamer & $-43.7_{2}$ & $-37.5_{5}$ & $-12.8_{6}$ & $-2.0_{2}$ \\
Solution & $-43.7_{1}$ & $-36.6_{6}$ & $-12.3_{7}$ & $-2.1_{2}$ \\
Surface 1 & $-42.6_{2}$ & $-31.0_{7}$ & $-6.9_{5}$ & $-1.0_{1}$ \\
Surface 2 & $-42.9_{3}$ & $-35.1_{7}$ & $-13.4_{10}$ & $-2.2_{2}$ \\
Surface 3 & $-44.1_{1}$ & $-37.9_{4}$ & $-14.7_{7}$ & $-2.9_{2}$ \\
Surface 4 & $-43.7_{2}$ & $-37.3_{7}$ & $-15.1_{12}$ & $-2.3_{3}$ \\
\hline Middle & $240 \mathrm{~K}$ & $260 \mathrm{~K}$ & $280 \mathrm{~K}$ & $300 \mathrm{~K}$ \\
\hline Hexamer & $-39.2_{2}$ & $-24.8_{5}$ & $-4.5_{3}$ & $-0.7_{1}$ \\
Solution & $-38.6_{2}$ & $-23.0_{5}$ & $-4.0_{3}$ & $-0.5_{1}$ \\
Surface 1 & $-38.0_{3}$ & $-20.6_{8}$ & $-3.7_{3}$ & $-0.6_{1}$ \\
Surface 2 & $-39.0_{2}$ & $-25.2_{8}$ & $-5.2_{5}$ & $-0.8_{1}$ \\
Surface 3 & $-37.1_{4}$ & $-20.3_{7}$ & $-3.4_{2}$ & $-0.5_{1}$ \\
Surface 4 & $-39.0_{3}$ & $-25.0_{10}$ & $-4.7_{4}$ & $-0.6_{1}$ \\
\hline Right & $240 \mathrm{~K}$ & $260 \mathrm{~K}$ & $280 \mathrm{~K}$ & $300 \mathrm{~K}$ \\
\hline Hexamer & $-38.4_{2}$ & $-23.2_{5}$ & $-4.4_{3}$ & $-0.6_{1}$ \\
Solution & $-38.6_{2}$ & $-24.0_{6}$ & $-5.3_{3}$ & $-0.8_{1}$ \\
Surface 1 & $-37.6_{4}$ & $-26.0_{6}$ & $-5.9_{4}$ & $-0.9_{1}$ \\
Surface 2 & $-38.1_{5}$ & $-26.2_{8}$ & $-7.0_{4}$ & $-1.1_{1}$ \\
Surface 4 & $-37.7_{4}$ & $-24.5_{6}$ & $-5.4_{4}$ & $-1.0_{1}$
\end{tabular}




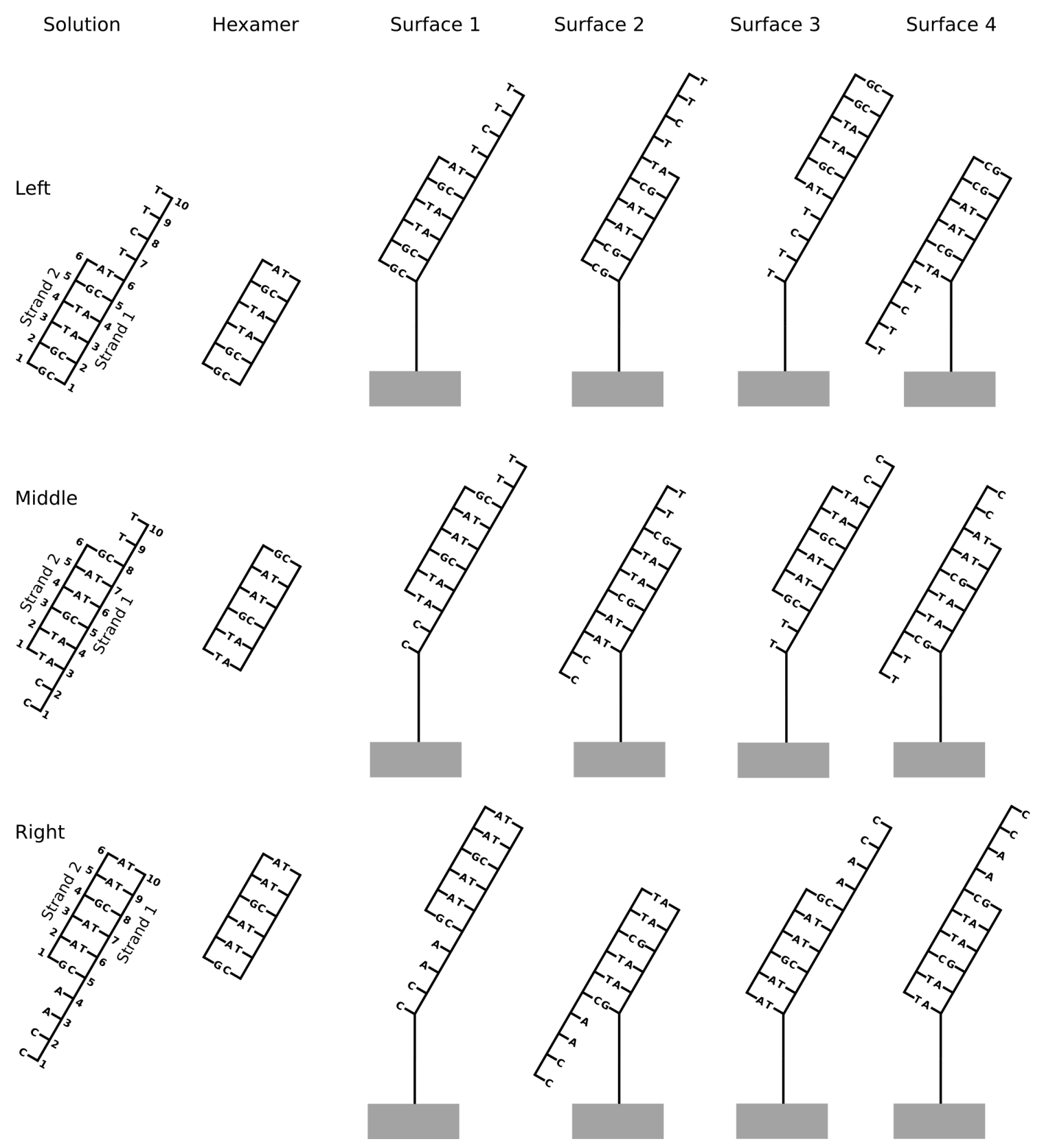

Figure 1: Illustration of all systems studied. 

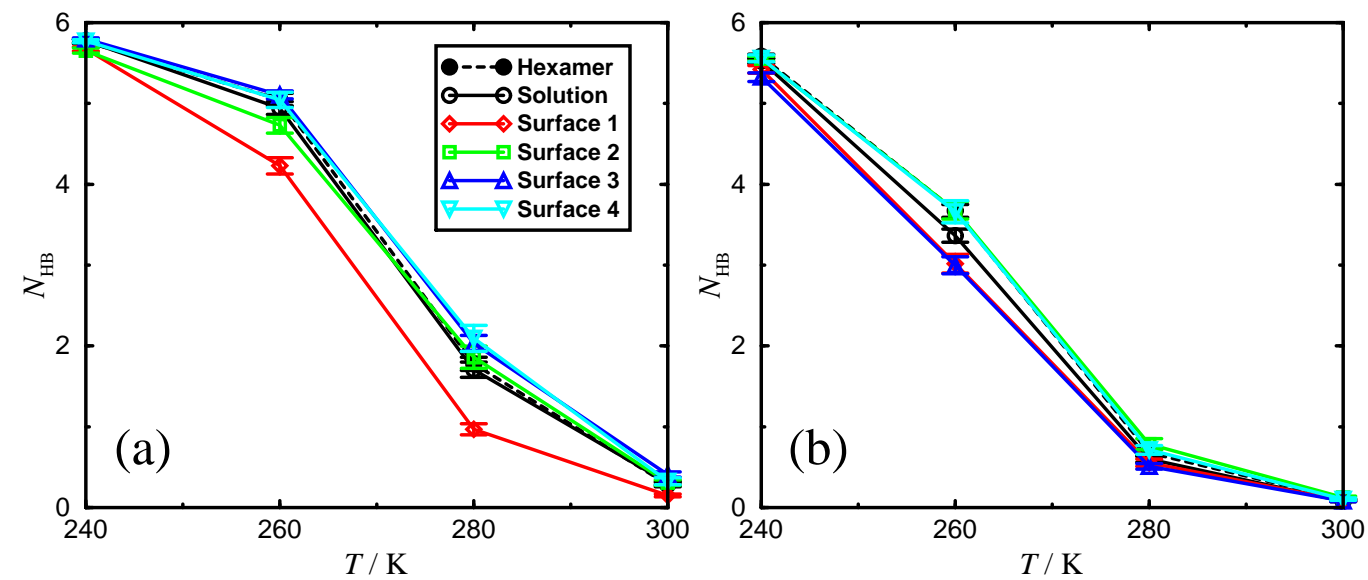

Figure 2: Average number of hydrogen bonding base pairs as a function of temperature for left and middle sequences in a) and b), respectively. Black filled circles, black open circles, red open diamonds, green open squares, blue open triangles up and cyan open triangles down (color online) correspond to hexamer, solution, surfaces 1, 2, 3 and 4, respectively. Lines are drawn as guides to the eye and dashed for the case of hexamer results.
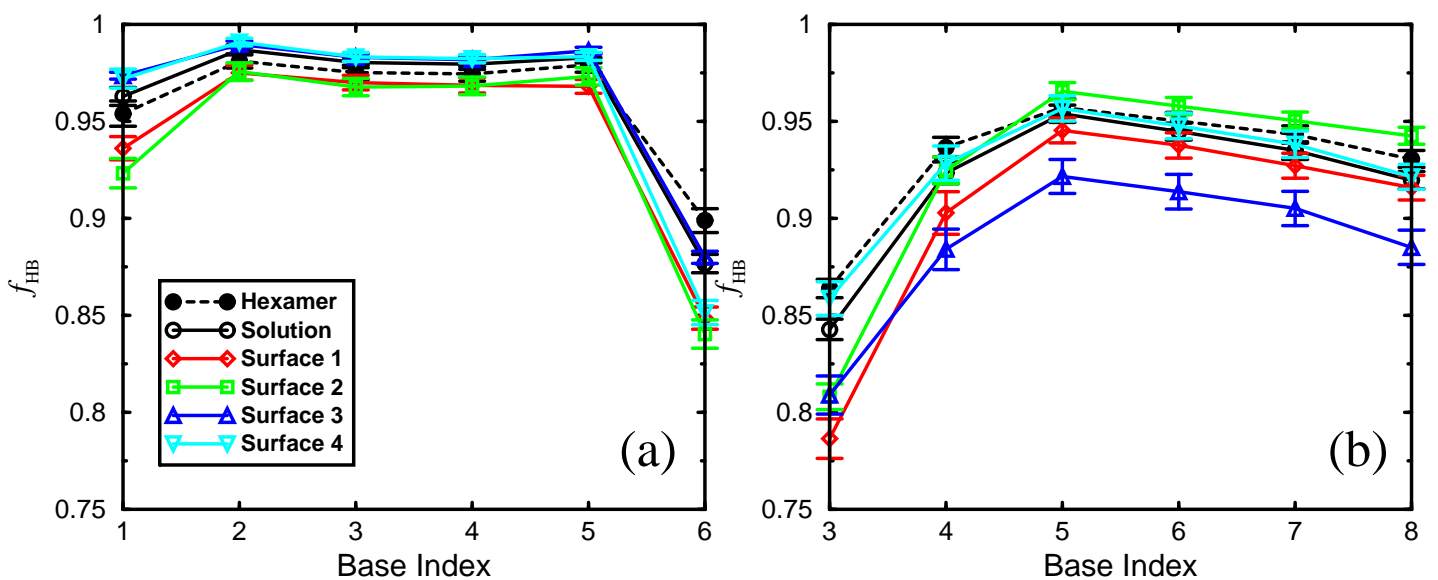

Figure 3: Fraction of hydrogen bonded configurations for a given base at $240 \mathrm{~K}$ for left and middle sequences in a) and b), respectively. Line styles as in Figure 2. 

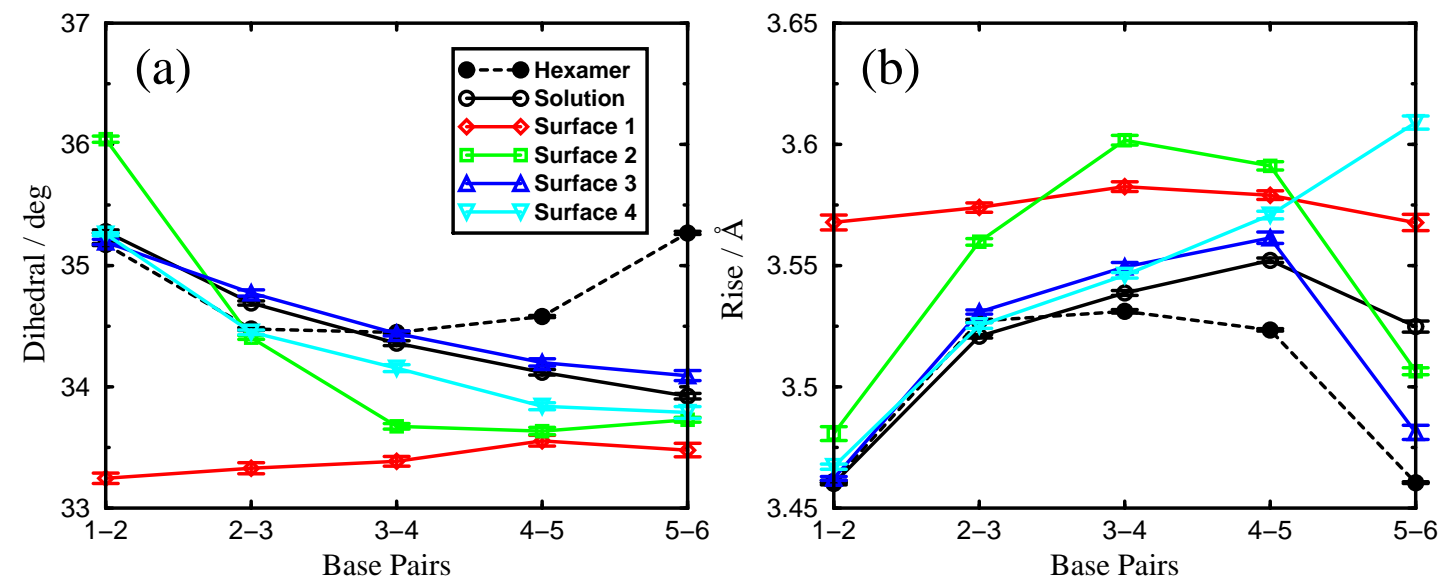

Figure 4: Base plane dihedral rotation (a) and rise distances (b) for the left sequence at 240 K. Line styles as in Figure 2.
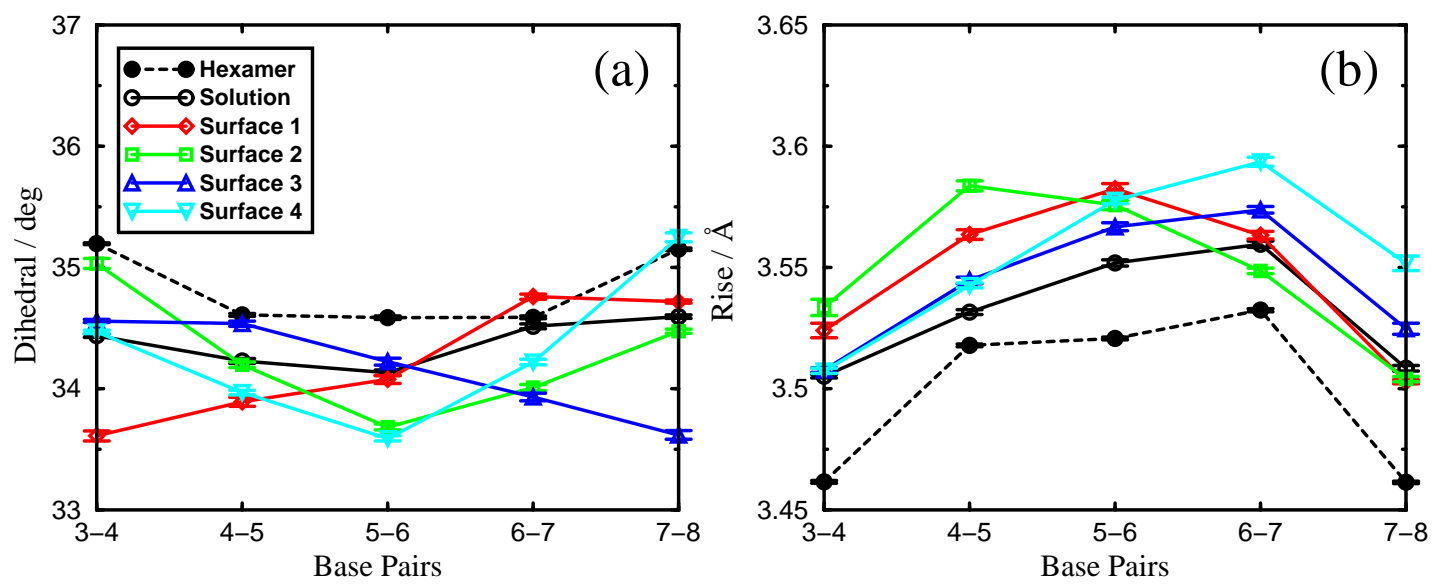

Figure 5: Base plane dihedral rotation (a) and rise distances (b) for the middle sequence at $240 \mathrm{~K}$. Line styles as in Figure 2. 


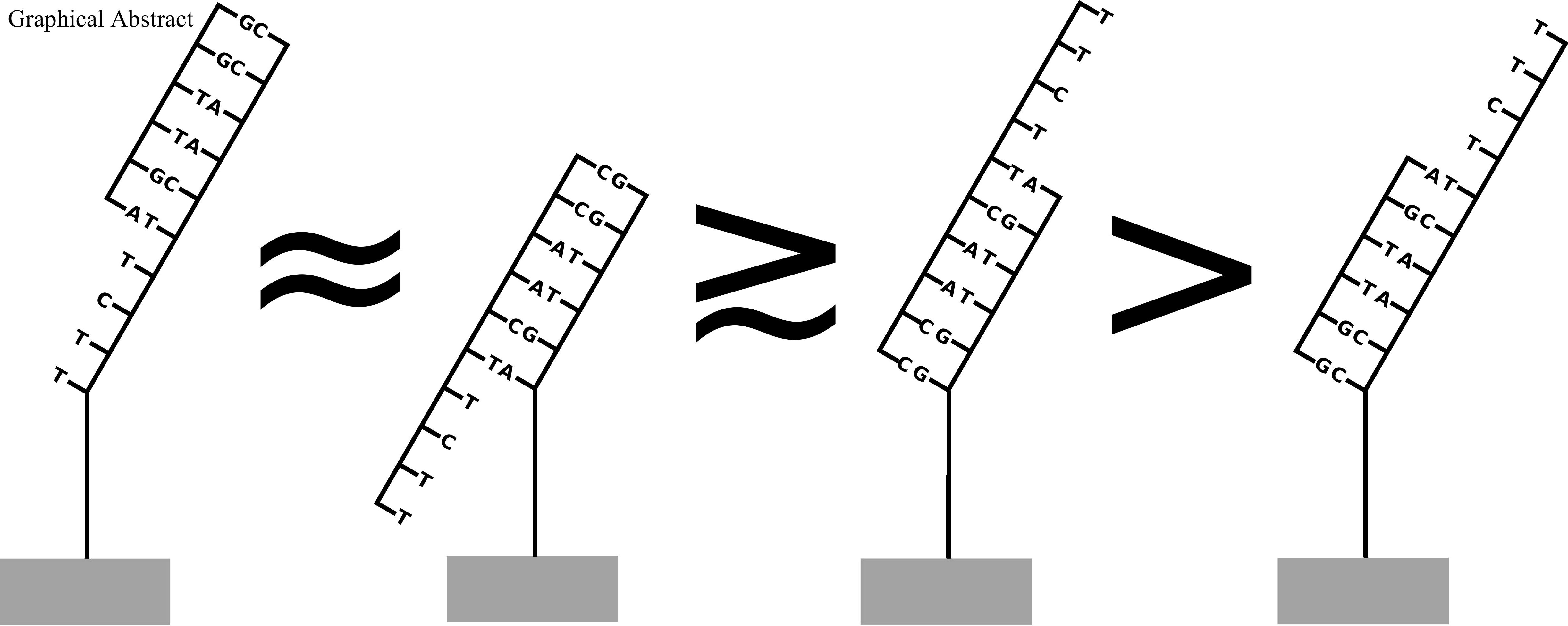

